\title{
Proton Synchrotron Radiation at Fermilab
}

\author{
Randy Thurman-Keup \\ Fermi National Accelerator Lab, P.O. Box 500, Batavia, IL 60510
}

\begin{abstract}
While protons are not generally associated with synchrotron radiation, they do emit visible light at high enough energies. This paper presents an overview of the use of synchrotron radiation in the Tevatron to measure transverse emittances and to monitor the amount of beam in the abort gap. The latter is necessary to ensure a clean abort and prevent quenches of the superconducting magnets and damage to the silicon detectors of the collider experiments.
\end{abstract}

Keywords: Synchrotron Radiation, Abort Gap, Transverse Profile

PACS: 07.85.Qe, 29.27.-a

\section{INTRODUCTION}

Synchrotron radiation is usually associated with electron accelerators since the intensity of the emitted radiation is proportional to $\gamma^{3}$. In the Tevatron at Fermilab, the $\gamma$ of the beam is $\sim 1000$ which is sufficient to obtain significant visible radiation from the superconducting dipole bend magnets. In 1979, R. Coisson [1] pointed out that the radiation emitted from "short" magnets and, more importantly for the Tevatron, magnet edges would have a frequency spectrum shifted toward the ultraviolet. This increase in the blue part of the spectrum for magnet edges has advantages for imaging the beam and enables measurements of the transverse beam sizes. The shorter wavelength means less diffraction, and since the shorter wavelength light is emitted predominantly from the edge of the magnet and not from the body of the magnet, the source is localized longitudinally. Figure 1 shows the dependence of intensity as a function of proton energy and optical wavelength.

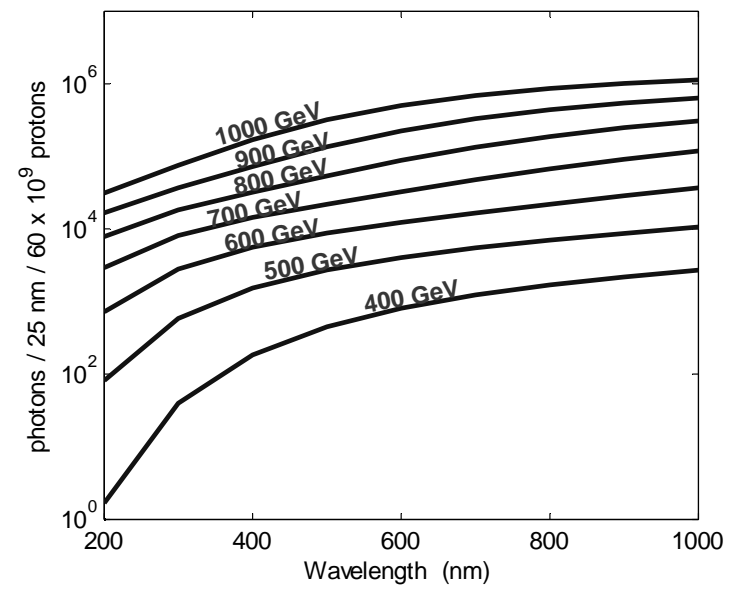

FIGURE 1. Energy and wavelength dependence of the intensity of synchrotron radiation emitted by protons near the edge of a magnet. 
A more critical use of synchrotron radiation at the Tevatron is for measuring the intensity of the DC component of the beam; in particular, the intensity of the DC beam located in the abort gap (see Fig. 2).

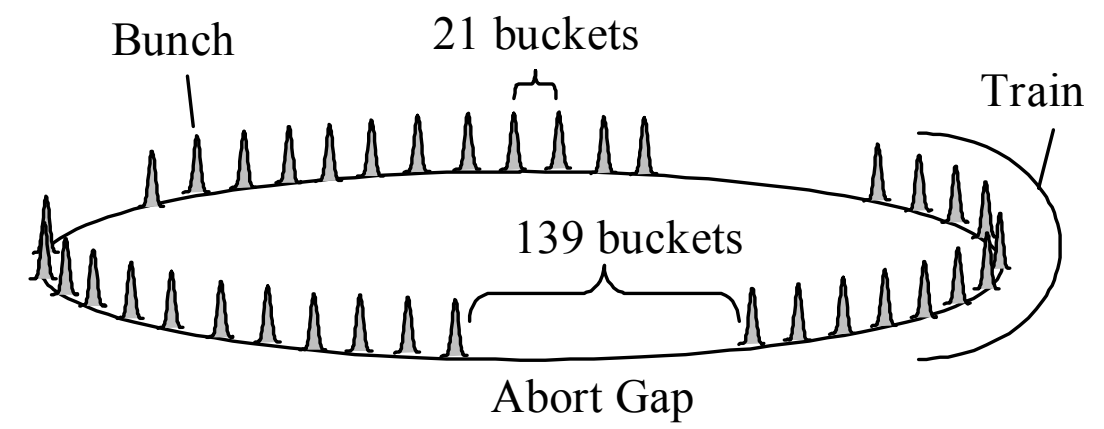

\section{RF buckets total $\quad$ RF bucket $=18.8 \mathrm{~ns}$}

FIGURE 2. Bunch layout of the Tevatron showing the relative locations of the abort gaps and the bunch trains.

During operation of the Tevatron, particles diffuse out of bunches and migrate forward. Because energy loss from synchrotron radiation is relatively small for protons, the migration continues for several minutes until the particles are intercepted by collimators. This migrating DC beam goes everywhere including the abort gaps. When beam is aborted, the abort kicker magnets ramp up during the abort gap. Beam that is present during that time doesn't receive the full kick and ends up producing large losses with devastating effects: quenches in the superconducting magnets, and radiation death for the silicon detectors of the collider experiments. To reduce the DC beam, the Tevatron uses a beam of electrons (a.k.a. TEL) which serves to eject particles from the abort gaps. Visible synchrotron radiation is a natural choice for monitoring the remaining level of DC beam since light is not susceptible to electronic noise, and optical detection devices with gains greater than $10^{6}$ are common. Previously, a measurement of the DC beam utilized a set of counters surrounding the beamline and gated in time with the abort gap. The problem with that approach is that it measures the amount of beam leaving, not the amount remaining.

\section{APPARATUS}

Figure 3 is a schematic of the synchrotron radiation pickoff device and its position relative to the adjacent Tevatron dipole magnets. It consists of a moveable mirror that is inserted into the beampipe and reflects the light out through a quartz vacuum window to a light-tight box. There are two of these pickoff mirror systems, one facing the proton direction and one facing the antiproton direction. In each box is a single plano-convex lens for focusing the light to a camera imaging system, and a beam splitter for dividing the light between the camera system and a gated photomultiplier tube (PMT). 


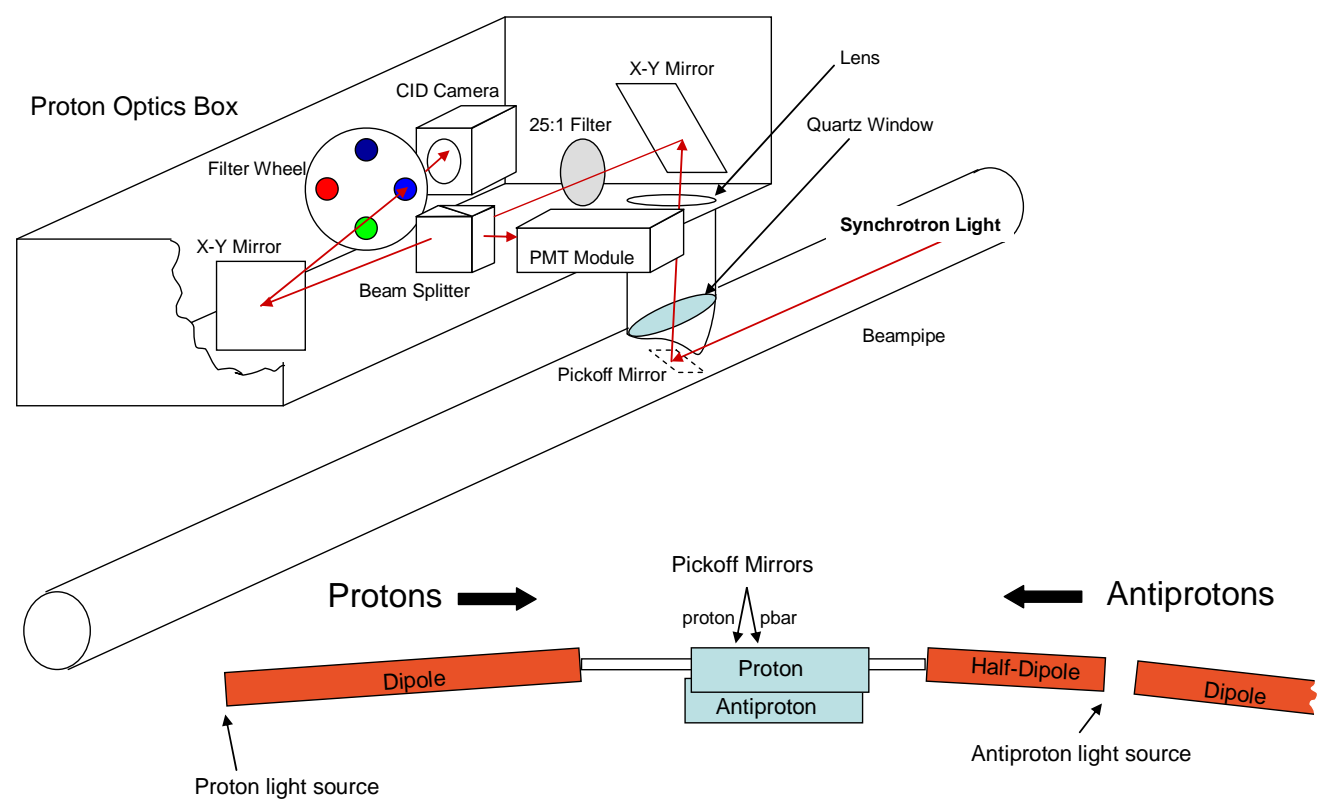

FIGURE 3. Top) Synchrotron radiation device schematic showing the pickoff mirror, associated optics box, and PMT module. Bottom) Physical layout relative to the adjacent dipole magnets.

\section{Camera System}

The camera systems are designed to measure the transverse beam size for each bunch by imaging the beam using light from a dipole magnet edge. They contain narrowband optical filters for discriminating against magnet body light and image intensifiers mounted to CID cameras. The narrowband filters have centers/bandwidths of 400/40 for the antiproton light and 440/10 for the proton light. The differences between the two were to accommodate diffraction measurements in the proton system. The image intensifiers are Hamamatsu V6887U-02 with $6 \mu \mathrm{m}$ channels. The cameras are CID cameras with $11.5 \mu \mathrm{m} \times 11.5 \mu \mathrm{m}$ pixels from ThermoElectron, and are intrinsically more resistive to radiation damage than their CCD cousins.

The data acquisition system is controlled by Labview running on a WindowsXP PC. The camera outputs an RS-170 video signal which is fed to a PCI-based framegrabber card in the PC. The Labview code fits the data for the horizontal and vertical beam sizes and converts them to emittances. Table 1 gives a summary of signal levels for the camera system.

TABLE 1. Parameters for the CID camera systems.

\begin{tabular}{lcc}
\hline & Proton & Antiproton \\
\hline Typical Bunch Intensity & $250 \times 10^{9}$ & $50 \times 10^{9}$ \\
\# photons emitted / bunch / 10nm & $250,000 @ 440 \mathrm{~nm}$ & $100,000 @ 400 \mathrm{~nm}$ \\
\# photons hitting image intensifier & 34,000 & 100,000 \\
\hline
\end{tabular}




\section{Photomultiplier Tube System}

The photomultiplier system is designed to measure the low light levels generated by the DC beam while remaining insensitive to the large light levels in the bunches. This is not a trivial requirement, however, since photomultiplier tubes are not generally designed to be gated on and off, and the typical bunch intensity is $\sim 1000$ times the DC beam intensity. The original device featured a design whereby two dynodes of a PMT were held at the wrong voltage to shut off the amplification process (Fig. 4). When the PMT was gated on, the two dynodes were capacitively pulsed to the correct voltage. This design worked but had 2 shortcomings: there was sensitivity to large amounts of light present just before application of the gate (Fig. 5), and gating the dynodes caused transients in the anode that made the signal unusable for $\sim 150 \mathrm{~ns}$ after the start of the gate. Fortunately, the first shortcoming was not a problem for the bunch intensities at the Tevatron, and the system was used for several months until a high speed gated PMT on loan from LBNL was installed.

The gated PMT on loan from LBNL is a Hamamatsu R5916U-50 which is a microchannel plate PMT and has a minimum gate width of $5 \mathrm{~ns}$. It has no detectable sensitivity to light present before the gate and has an extinction ratio of $10^{7}$. Its drawbacks are a gain of only $5 \times 10^{5}$ and a gating duty cycle of only $1 \%$, both of which have been addressed in modified versions of the tube recently purchased and soon to be installed. Because of the narrow gating capability, measurements of the DC beam can be made between bunches as well as in the abort gaps.
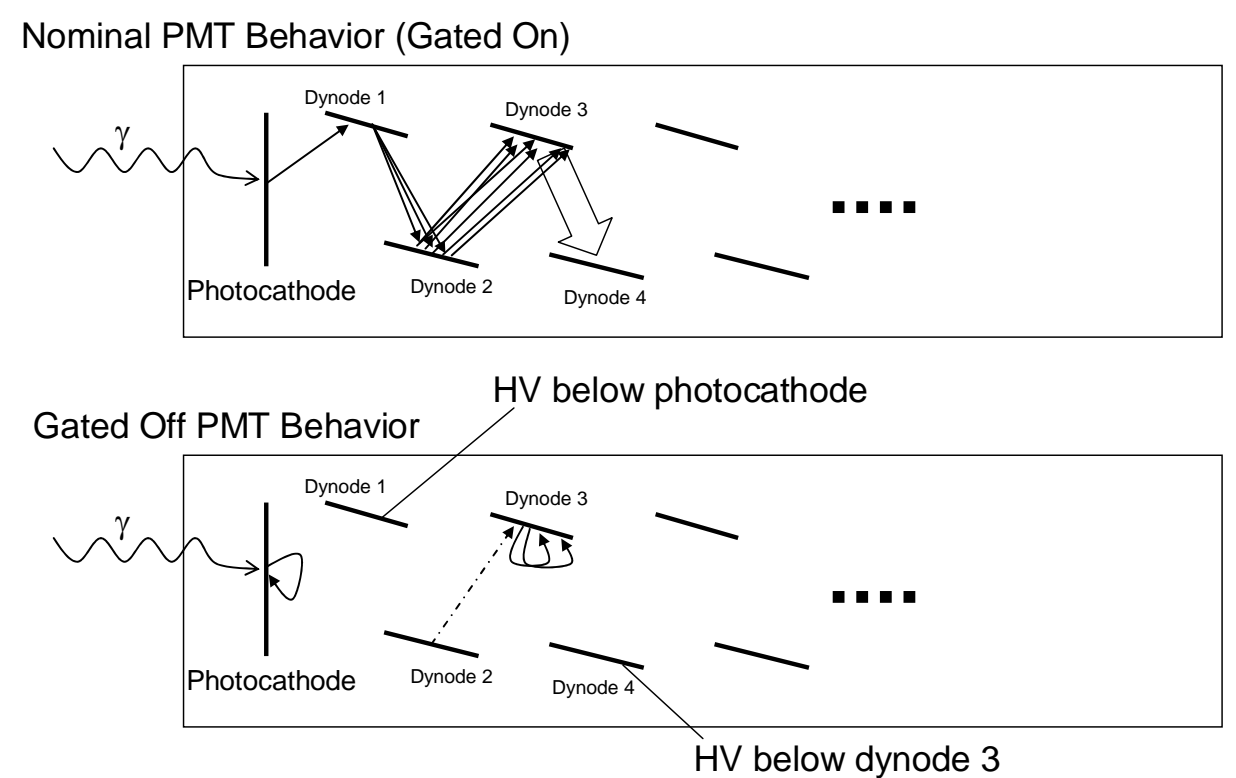

FIGURE 4. Design of a gated PMT whereby two dynodes are held at the wrong voltage, shutting off the amplification process. When gated on, the dynodes are pulsed to their nominal voltages, and the PMT functions normally. 


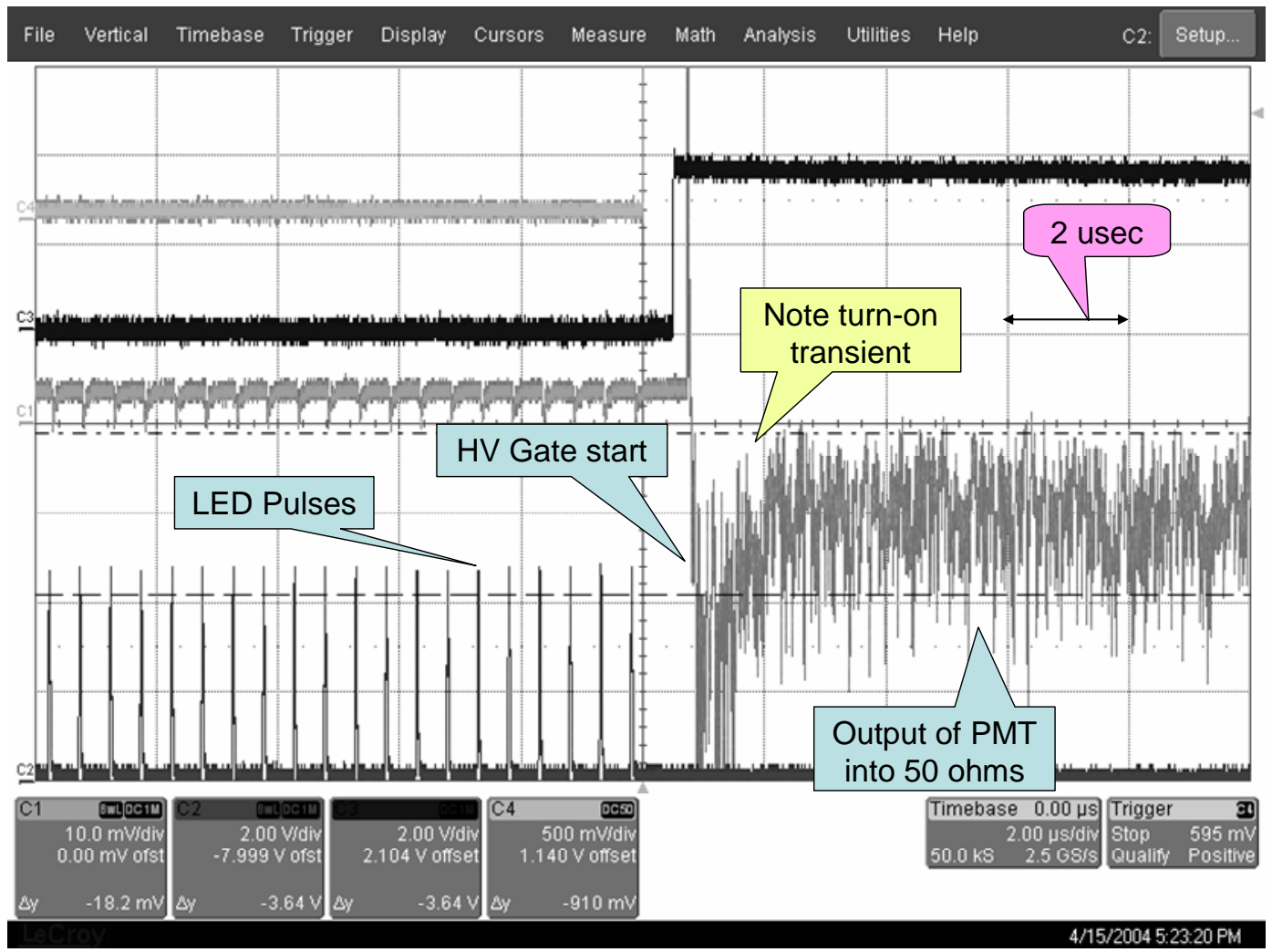

FIGURE 5. Response of the gated dynode PMT to simulated beam. There is a turn-on transient that is dependent on the amount of light present from the bunch just before the gate is applied.

The data acquisition system is controlled by an application running under VxWorks on a VME-based PowerPC processor board. The anode signal of the PMT is integrated, digitized, and averaged over 1000 turns to obtain an accurate measurement. The application controls the timing of the integration and digitization cycles. Table 2 gives a summary of signal levels for the PMT system.

TABLE 2. Parameters for the PMT system.

\begin{tabular}{lc}
\hline Total Beam Intensity & $10^{13}$ protons $/ 10^{12}$ antiprotons \\
Desired DC Beam Sensitivity & $10^{9}$ \\
\# DC Beam photons emitted / 25nm & 3 \\
/ RF Bucket & 420 \\
/ Abort Gap & \\
$100 \mathrm{~nm}$ bandwidth; 35\% optical losses & \\
\# photons hitting PMT & 4 \\
/ RF Bucket & 560 \\
\hline / Abort Gap & \\
\hline
\end{tabular}

\section{ABORT GAP INTENSITY CALIBRATION}

The calibration of the PMT system is accomplished in two ways: direct comparison with bunch intensity measurements using RF pickups, and utilization of the TEL to produce a change in the total beam intensity as measured by a DC current transformer 
(DCCT). Comparison with the RF pickup measurement is straightforward. Insert optical attenuators into the light path and measure the intensity of a bunch. This relies on knowing the attenuation factor and the linearity of the PMT. The second method is more unique and doesn't involve changing the setup of the PMT system. Under normal operations, the TEL is active during the abort gaps to keep the DC beam level to a minimum. If the TEL is turned off, DC beam accumulates until it reaches some equilibrium. The DCCT is accurate to 1 part in $10^{5}$ and as such can see changes in the total beam of $\sim 10^{8}$. When the TEL turns off, the rate of change of the beam intensity decreases since beam is no longer being ejected by the TEL (Fig. 6).

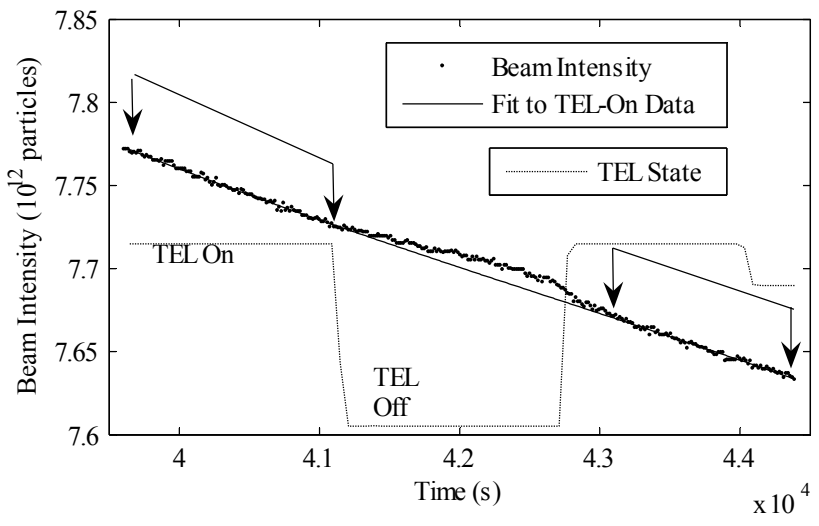

FIGURE 6. Behavior of total beam intensity as the TEL is turned off and back on. The arrows indicate the regions that are fit with a quadratic to obtain the TEL-on baseline.

Extrapolating from before the TEL turned off, the difference between that and the DCCT value is the extra DC beam that has accumulated. This value can be compared with the measurement by the PMT system to obtain a calibration value (Fig. 7).

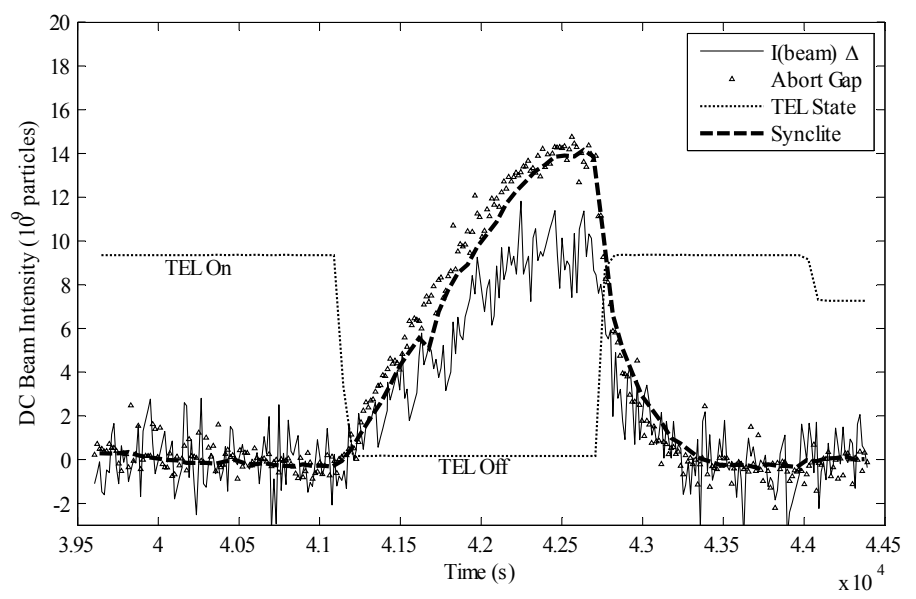

FIGURE 7. The baseline-subtracted total beam intensity, labeled I(beam) $\Delta$ in the plot, is compared to baseline-subtracted measurements from the PMT in the abort gap and to the CID camera system (a.k.a. Synclite).

The disadvantage of this approach is that it takes a special study request to turn off the TEL. The best approach is to use a combination of the two calibration methods 
where the TEL version is occasionally done to obtain an absolute calibration, and the bunch intensity comparison is used to track changes in the calibration between TEL measurements.

\section{RESULTS}

Figure 8 is an image of the beam taken with the CID camera system. Measurements of the emittance produced with this system agree with that produced by the Flying Wire system to better than $10 \%$. More studies are still in progress to understand the remaining discrepancies.
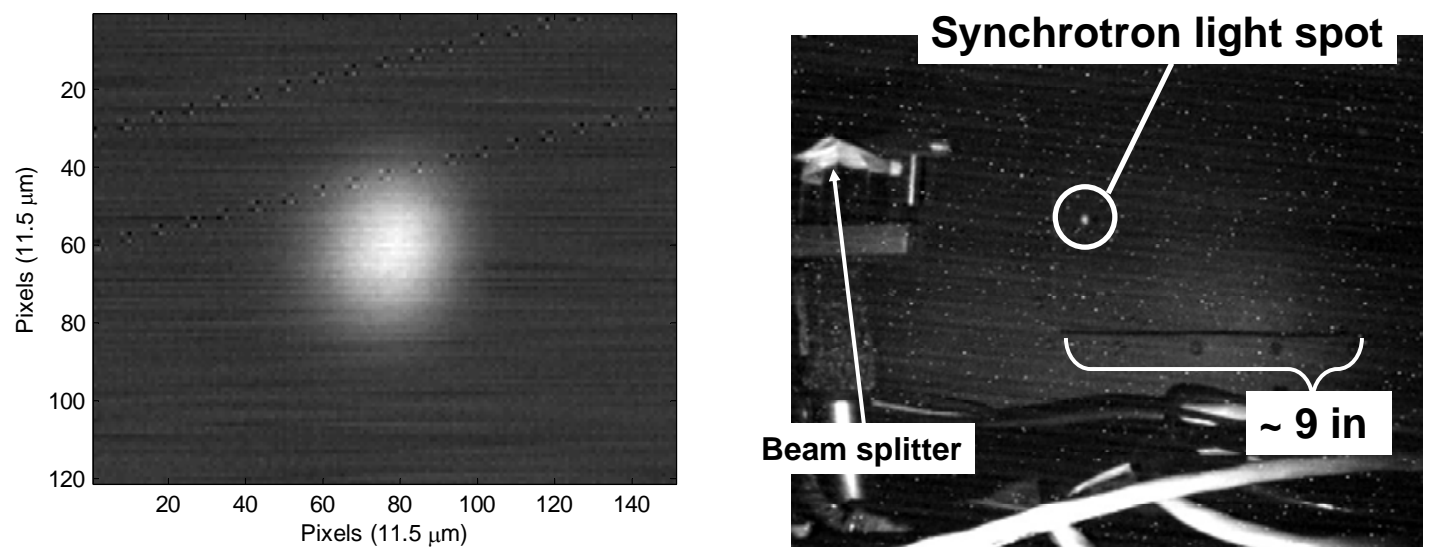

FIGURE 8. Left) Typical image of the proton beam taken with the CID camera system. Right) Partially focused synchrotron light spot projected on the wall of the optics box.

The abort gap PMT system has been in use for almost 2 years. It is incorporated into the end of beam sequence where it is checked to be below some limit before beam can be dropped. The CDF collider experiment monitors it regularly as part of their silicon detector safety protocol. Figure 9 demonstrates the normal behavior of the system at the beginning of a store. Several features related to TEL operation can be seen. 

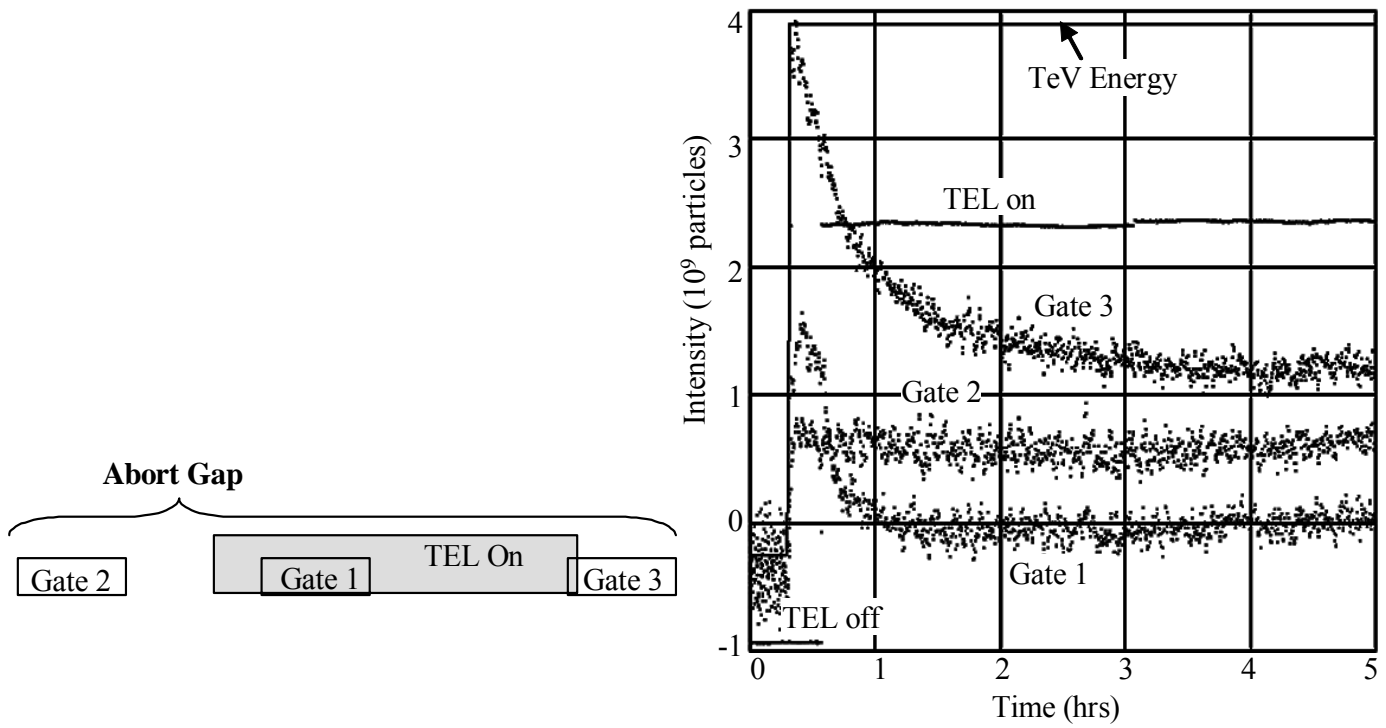

FIGURE 9. Typical abort gap beam intensity behavior at the beginning of a store. The 3 gates are at different positions in the 3 gaps. The TEL turns on $\sim 15$ minutes after the ramp to $980 \mathrm{GeV}$. At that time, gate 1, which overlaps the TEL, is cleared out. The beam that is in the abort gap after ramping is a captured remnant left over from coalescing. The diagram adjacent to the plot shows the relative timing of the gates and the TEL.

Figure 10 is an example of the behavior of the system when the longitudinal dampers were misfiring and the beam was shaken and spread out around the ring. The behavior of the three positions in the abort gap is consistent with the behavior at the start of a store (see Fig. 9).

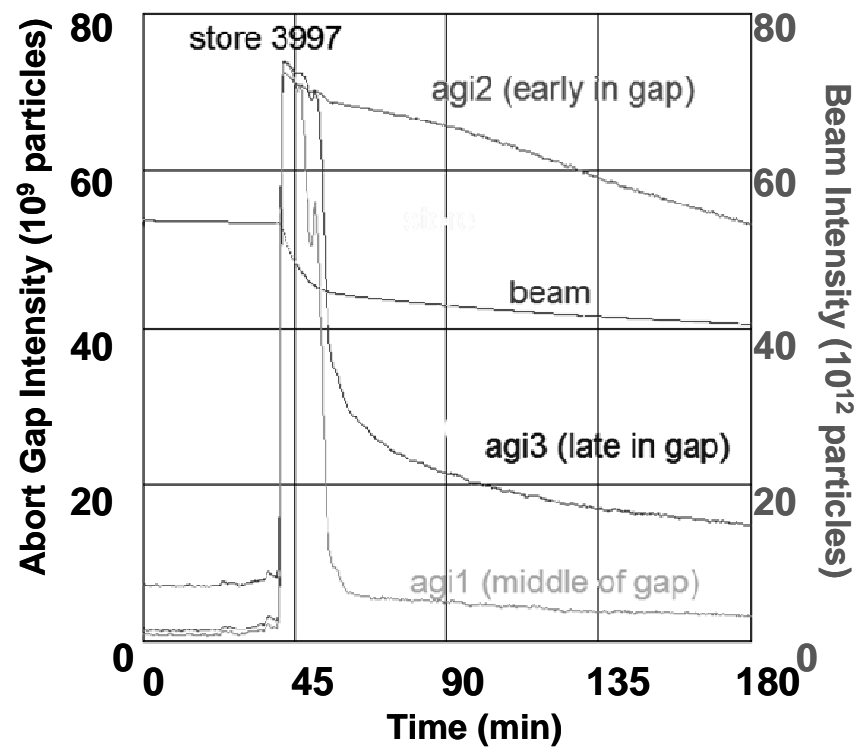

FIGURE 10. The result of the longitudinal dampers losing their mind. Beam is shaken out of bunches and spread around the ring. Just before the 45-minute mark, there is a loss of beam and the abort gap PMT saturates. After some number of minutes, the dampers settle down and the TEL starts clearing the abort gaps. 
Since the gate width of the PMT can be a single RF bucket, the behavior of the DC beam between bunches can be studied. Figure 11 shows the change in DC beam in the RF buckets between two bunches over the course of a store.

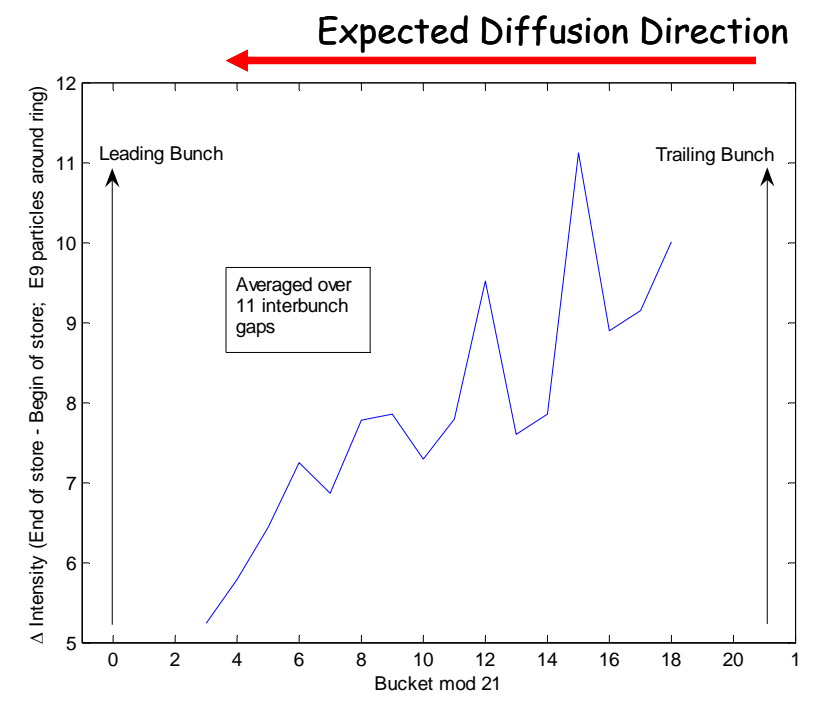

FIGURE 11. The difference in DC beam from beginning to end of store as a function of bucket number between two bunches. This is an average over all the bunch gaps in a train of 12 .

Another interesting measurement is to use the PMT in photon counting mode and determine the structure of the beam in the abort gap. Figure 12 examines the structure near the end of the abort gap during a time when there was significant beam there.

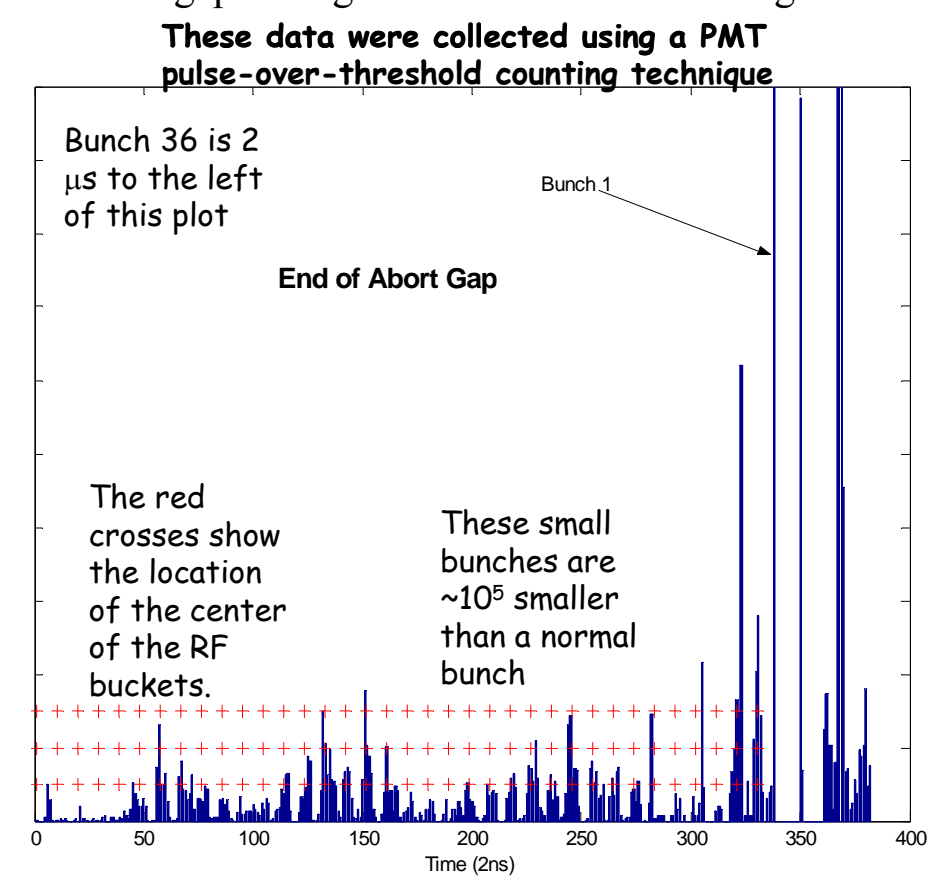

FIGURE 12. Plot showing the existence of microbunches near the end of the abort gap. If they are captured in RF buckets, the peaks should be centered on the red crosses. Since the peaks are in the center of the RF buckets, they are probably left over remnants from coalescing in the Main Injector. 


\section{SUMMARY}

Synchrotron radiation has found a number of uses at the Tevatron. It is used to make transverse profile measurements and has become an indispensable tool for preventing superconducting magnet quenches and severe radiation damage to the silicon detectors of the collider experiments.

\section{ACKNOWLEDGMENTS}

I want to thank Stephen Pordes, Tom Meyer, Eugene Lorman, Carl Lundberg, Dale Miller, Alex Valishev, Alan Hahn, Harry Cheung, Vladimir Shiltsev, and many others for all their contributions to the construction and operation of the synchrotron light devices at the Tevatron. This work was supported by the United States Department of Energy.

\section{REFERENCES}

1. R. Coïsson, Angular-Spectral Distribution and Polarization of Synchrotron Radiation from a "Short" Magnet, Phys. Rev. A20 (1979) 2. 